\title{
The Effect of Temperature on Synthesis and Stability of Superparamagnetic Maghemite Nanoparticles Suspension
}

\author{
Irwan Nurdin*, Ridwan, Satriananda \\ Department of Chemical Engineering, Lhokseumawe State Polytechnic, Lhokseumawe, Indonesia \\ Email: *irwan_nurdina@yahoo.com
}

Received 19 February 2016; accepted 22 March 2016; published 25 March 2016

Copyright ( 2016 by authors and Scientific Research Publishing Inc.

This work is licensed under the Creative Commons Attribution International License (CC BY).

http://creativecommons.org/licenses/by/4.0/

c) (i) Open Access

\begin{abstract}
Maghemite $\left(\gamma-\mathrm{Fe}_{2} \mathrm{O}_{3}\right)$ nanoparticles have been synthesized using chemical co-precipitation at a different temperature. Characterizations of the sample were performed by X-ray diffraction (XRD), transmission electron microscopy (TEM), alternating gradient magnetometry (AGM) and thermogravimetryanalysis (TGA). The stability of the maghemite nanoparticles suspension was studied at different $\mathrm{pH}$ and time of storage by dynamic light scattering (DLS) and zeta potential measurements. The XRD patterns confirmed that the particles were maghemite. TEM observation showed that the particles have spherical morphology with narrow particle size distribution. The particles showed superparamagnetic behavior with good thermal stability. The increasing of temperature in the synthesis of maghemite nanoparticles produced smaller size particles, lower magnetization, better thermal stability and more stable maghemite nanoparticle suspension.
\end{abstract}

\section{Keywords}

Temperature Effect, Synthesis, Maghemite, Nanoparticles, Characterization

\section{Introduction}

Due to their unique behaviors, many researchers have been interested in magnetic nanoparticles in recent years. They are using in a broad range of applications including electronic, mechanical engineering, aerospace, environmental and bioengineering [1]-[3]. Some of the particular applications of these magnetic nanoparticles are in fields of micro-electronics as the audio speaker, energy supply, HVAC, magnetic seal in motors, magnetic ink for banking cheques. They also used as magnetic recording media, magnetic resonance imaging media, drug de-

\footnotetext{
${ }^{*}$ Corresponding author.

How to cite this paper: Nurdin, I., Ridwan and Satriananda (2016) The Effect of Temperature on Synthesis and Stability of Superparamagnetic Maghemite Nanoparticles Suspension. Journal of Materials Science and Chemical Engineering, 4, 35-41. http://dx.doi.org/10.4236/msce.2016.43005
} 
livery or therapeutic agents in cancer therapy and thermal engineering application [4]-[8]. Iron oxide nanoparticles, mainly magnetite $\left(\mathrm{Fe}_{3} \mathrm{O}_{4}\right)$ and maghemite $\left(\gamma-\mathrm{Fe}_{2} \mathrm{O}_{3}\right)$, are favorable magnetic materials that are intensively explored due to their specific magnetic properties [9]. The suspensions of these magnetic nanoparticles in a liquid medium generate a new class of liquid called "magnetic fluids."

The uniqueness of this fluid is its superparamagnetic and biocompatibility behavior, and it can be controlled by external magnetic fields. Hence, the magnetic fluids also can be effectively used in thermal engineering applications and carrier media [7].

Many efforts have been made to synthesize stable magnetic suspension in recent years to accomplish appropriate control of magnetic particle size, shape, crystallinity, and magnetic properties [10]. Numerous techniques have been reported on the synthesis of magnetic nanoparticles. They included chemical co-precipitation [11] [12], sol-gel synthesis [13] [14], microemulsion [15]-[17], thermal decomposition [18] [19], and hydrothermal [20] [21]. The most simple and common technique is co-precipitation [22] [23]. This method is reproducible, simple and cheap, and it gives high yields result. Most of the researchers reported the synthesis of magnetite nanoparticles rather than maghemite nanoparticles.

Even many efforts have been made towards the synthesis of stable magnetic nanoparticles suspension, it still presents a significant challenge. The stability of the magnetic nanoparticles suspension is the most important parameter in the preparation of this material. In this paper, maghemite nanoparticles were synthesized by chemical co-precipitation method at various temperatures and characterized by a numerous of analytical techniques. The stability of maghemite nanoparticles suspensions was evaluated by observing their particle size distribution with dynamic light scattering (DLS) and zeta potential measurements.

\section{Material and Methods}

The chemical reagents used in this research were ferric chloride hexahydrate $\left(\mathrm{FeCl}_{3} \cdot 6 \mathrm{H}_{2} \mathrm{O}\right)$, ferrous chloride tetrahydrate $\left(\mathrm{FeCl}_{2} \cdot 4 \mathrm{H}_{2} \mathrm{O}\right)$, and ferric nitrate nonahydrate $\left(\mathrm{Fe}\left(\mathrm{NO}_{3}\right)_{3} \cdot 9 \mathrm{H}_{2} \mathrm{O}\right)$. These chemicals were procured from Sigma-Aldrich. Ammonium hydroxide $\left(\mathrm{NH}_{4} \mathrm{OH}\right) 28 \%$, nitric acid $\left(\mathrm{HNO}_{3}\right) 65 \%$, and hydrochloric acid $(\mathrm{HCl})$ $37 \%$ were purchased from Merck Chemical. The solvent used for all the experiments were deionized water with the resistivity of around $15 \mathrm{M} \Omega / \mathrm{cm}$. All reagents were analytical grade and were used as received without further purification. Five samples were prepared with various temperatures, labeled as S50, S60, S70, S80, and S90 for temperatures of $50^{\circ} \mathrm{C}, 60^{\circ} \mathrm{C}, 70^{\circ} \mathrm{C}, 8^{\circ} \mathrm{C}$, and $90^{\circ} \mathrm{C}$, respectively.

The solutions of ferric chloride and ferrous chloride using molar ratio 2:1 were mixed. Then, ammonium hydroxide solution was added to the solution with vigorous stirring at $270 \mathrm{rpm}$ using a Wise Stir direct driven stirrer HS-50A for 20 minutes at room temperature. A black precipitate formed immediately and separated from the solution and washed several times by deionized water. The precipitate was then stirred for 10 minutes in $10 \mathrm{M}$ nitric acid solution. The precipitate washed several times and then oxidized to maghemite at various temperatures ranging from $50^{\circ} \mathrm{C}$ to $90^{\circ} \mathrm{C}$ for 30 minutes using ferric nitrate solution. Brown precipitate was isolated from solution and then washed and peptized thoroughly with deionized water.

X-ray powder diffraction (XRD) measurements were conducted using a Philips X'Pert MPD X-Ray Diffractometer, with copper source $(\lambda=1.54056 \AA)$ with a scan range of $20^{\circ}-80^{\circ} 2 \theta$ angle at a step of $0.05^{\circ}$ and a count time of $5 \mathrm{~s}$ at each step. The morphology and physical size of the particles were observed using transmission electron microscopy (TEM). The images were taken using a Leo LIBRA transmission electron microscope operated at $120 \mathrm{kV}$. The magnetic property of the maghemite was measured by an Alternating Gradient Magnetometer (MicroMag, model 2900) with applied fields of $\pm 10 \mathrm{kOe}$ at room temperature. Thermal gravimetry analysis (TGA) was performed to investigate the thermal behavior of the samples. TGA analysis was performed from ambient temperature to $1000^{\circ} \mathrm{C}$ with a heating rate of $10^{\circ} \mathrm{C} / \mathrm{min}$. The hydrodynamic diameter and zeta potential of the nanoparticles suspension were determined by dynamic light scattering (DLS) using a Malvern Zetasizer $3000 \mathrm{HS}$ at $25^{\circ} \mathrm{C}$.

\section{Results and Discussion}

\subsection{Maghemite Nanoparticles Characterization}

The XRD patterns of all samples are shown in Figure 1. The patterns display well-defined peaks that clearly indicate the crystallinity of the samples. The reflection peaks in the pattern were indexed to face center cubic (fcc) 


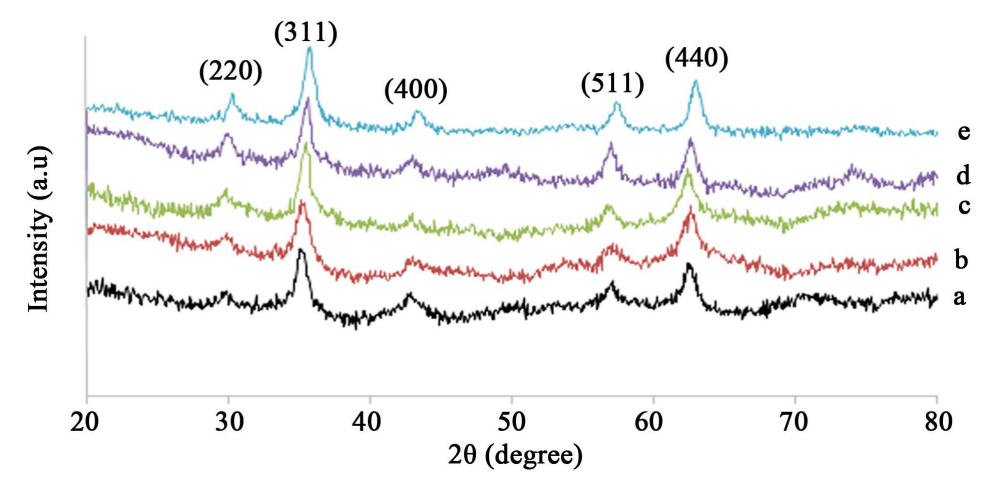

Figure 1. XRD patterns of maghemite nanoparticles for samples: (a) S50; (b) S60; (c) S70; (d) S80; and (e) S90.

phase with lattice parameters ranging from 8.334 to $8.368 \AA$. These lattice parameters are in good agreement with the bulk lattice parameter of maghemite $(a=8.3474 \AA)$ [24]. The crystallite size of the nanoparticles was calculated from the XRD line broadening using Scherrer's equation. The crystallite sizes are 16.2, 15.4, 13.7, 11.6, and $10.3 \mathrm{~nm}$ for S50, S60, S70, S80, and S90 samples, respectively. It is shown that the crystallite sizes of nanoparticles are gradually reduced if the temperature is rises.

The shape and particle size distribution of maghemite nanoparticles were observed by transmission electron microscopy (TEM) as shown in Figure 2. It is clearly shown that the maghemite particles have a spheroidal shape. The sizes of the particles were measured from about 100 particles. The particle sizes are 17.8, 16.9, 15.6, 12.4, and $9.6 \mathrm{~nm}$ for S50, S60, S70, S80, and S90 samples, respectively. There are a little bigger 'particles' which are created to be aggregates. It may be due to long-range magnetic dipole-dipole interaction between the particles. This average physical size is in good relationship with the crystallite size achieved from XRD measurement indicating that the particles are largely monocrystals.

The magnetization curves for all samples are shown in Figure 3. It is clear that the magnetization curves do not display coercivity and remanence which indicates that the samples are superparamagnetic. The saturation magnetization values of maghemite nanoparticles at room temperature for all samples are 34.3, 32.2, 30.8, 27.5, and $25.5 \mathrm{emu} / \mathrm{g}$ for S50, S60, S70, S80, and S90 samples, respectively. These values are lower than that of bulk maghemite $(74 \mathrm{emu} / \mathrm{g})$ due to the crystallite size of maghemite particles are in nanosize range. This phenomenon is usually happened in nanoparticles interacting systems. The decrease of magnetization can be ascribed to surface effects arising from broken symmetry and reduced synchronization of atoms lying at the surface of maghemite nanoparticles. Moreover, it also caused by a high degree of interparticle interactions [25].

TGA curves of the maghemite nanoparticles at different temperatures are shown in Figure 4. It can be seen that the curves exhibit similar weight loss behavior and display two weight losses steps. The initial weight loss starts from the ambient temperature to $200^{\circ} \mathrm{C}$ and the final weight loss is within the temperature range of 210 to $450^{\circ} \mathrm{C}$. The initial weight loss is associated with the evaporation of absorbed water and crystalline water from the sample. The final weight loss might be attributed to the volatilization of the remainder bonding water in the sample which will evaporate at water critical temperature of $374^{\circ} \mathrm{C}$. No further significant weight loss or gain is found in the temperature range of $450^{\circ} \mathrm{C}$ to $1000^{\circ} \mathrm{C}$, indicating crystalline of maghemite has been formed entirely.

The temperature stability $\left(\mathrm{T}_{\mathrm{s}}\right)$ for all samples when maghemite completely formed are $530^{\circ} \mathrm{C}, 515^{\circ} \mathrm{C}, 505^{\circ} \mathrm{C}$, $490^{\circ} \mathrm{C}$, and $460^{\circ} \mathrm{C}$ for S50, S60, S70, S80, and S90 samples, respectively. It can be seen that the temperature stability decreases with increasing temperature of synthesis. This indicate that sample with the higher temperature is stabilized earlier than other samples.

\subsection{Stability Characterization}

The particle size distributions of maghemite nanoparticles suspension achieved from dynamic light scattering (DLS) measurement are shown in Figure 5. The particles sizes are 193.5, 130.9, 105.2, 77.2 and $58.2 \mathrm{~nm}$ for S50, S60, S70, S80, and S90 samples, respectively. It indicated that the particles size decrease with the increasing of temperature. It is also displayed that the particle sizes obtained are larger than the TEM results. The DLS 


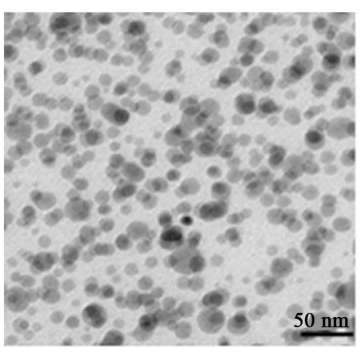

(a)

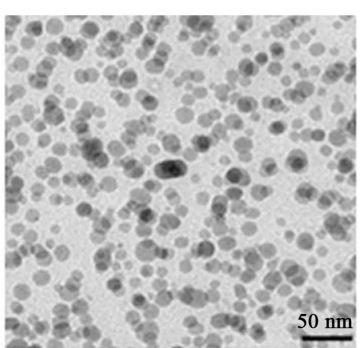

(b)

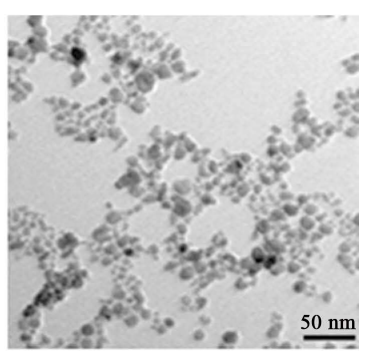

(c)

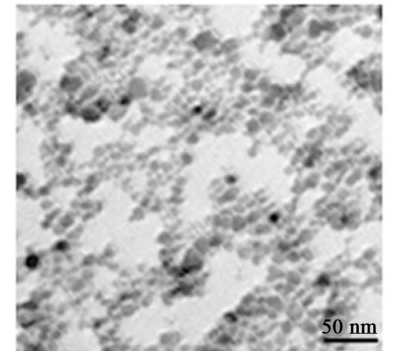

(d)

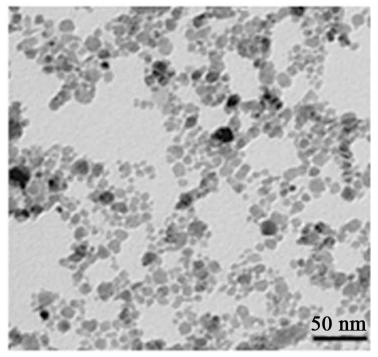

(e)

Figure 2. TEM images of maghemite nanoparticles for samples: (a) S50; (b) S60; (c) S70; (d) S80; and (e) S90.

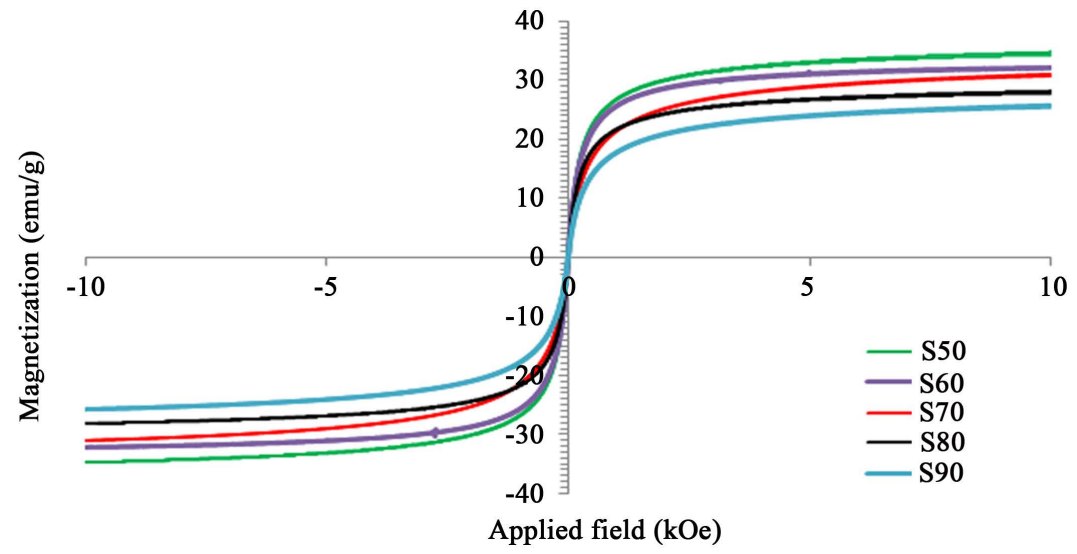

Figure 3. Magnetization curves of maghemite nanoparticles for samples.

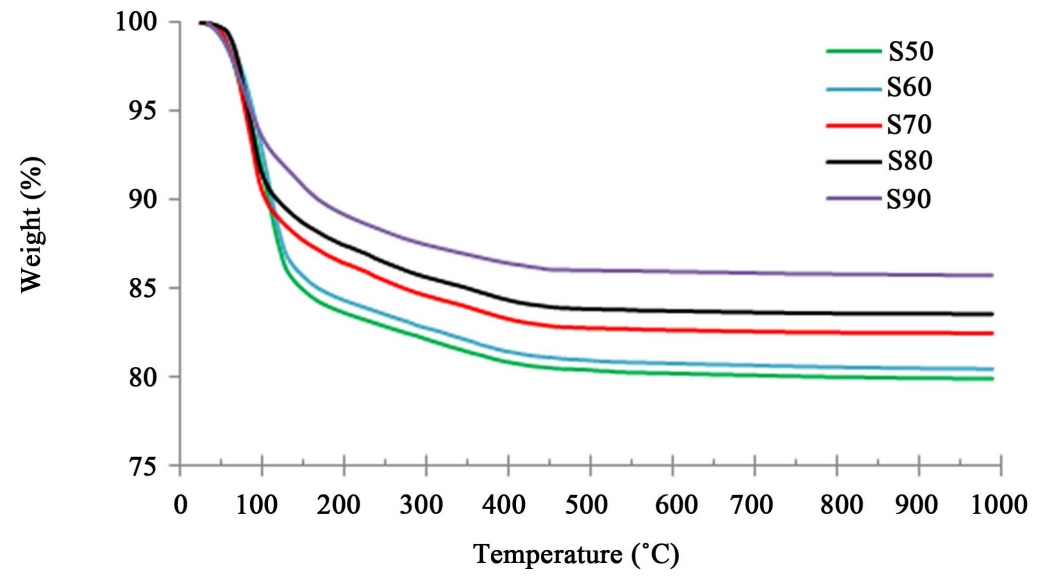

Figure 4. TGA thermograms of maghemite nanoparticles for samples. 
measure the hydrodynamic diameter of the particles in the suspensions, which is the diameter of the particles and their surrounding layer, while TEM measure the physical size of the particles themselves.

Since the stability of suspension is related to its electrokinetics properties, therefore, the study of electrophoretic behavior through measurement of zeta potential becomes compulsory for understanding the stability of suspension [26]. It is recognized that nanoparticles suspensions become stable with a zeta potential value higher than $\pm 30 \mathrm{mV}$. The zeta potentials of maghemite nanoparticles suspension are shown in Figure 6 . The values of zeta potentials are 30.1, 31.2, 36.3, 39.6, and $41.7 \mathrm{mV}$ for S50, S60, S70, S80, and S90 samples, respectively. These values indicate that the maghemite nanoparticles suspensions are stable.

\section{Conclusion}

Stable maghemite nanoparticles have been successfully synthesized by co-precipitation method at various temperatures. The patterns obtained from XRD show well define peaks which clearly indicate that the samples are crystalline. They also reveal that the particles are confirmed maghemite. TEM observations and image analysis show that the maghemite nanoparticles have the spherical morphology and small size particles. Magnetization curves show that maghemite nanoparticles exhibit superparamagnetic behavior. The particles show good thermal stability during thermogravimetry analysis. The increasing temperatures in the synthesis of maghemite nanoparticles can produce smaller size, lower magnetization, better thermal properties, and more stable maghemite nanoparticles.

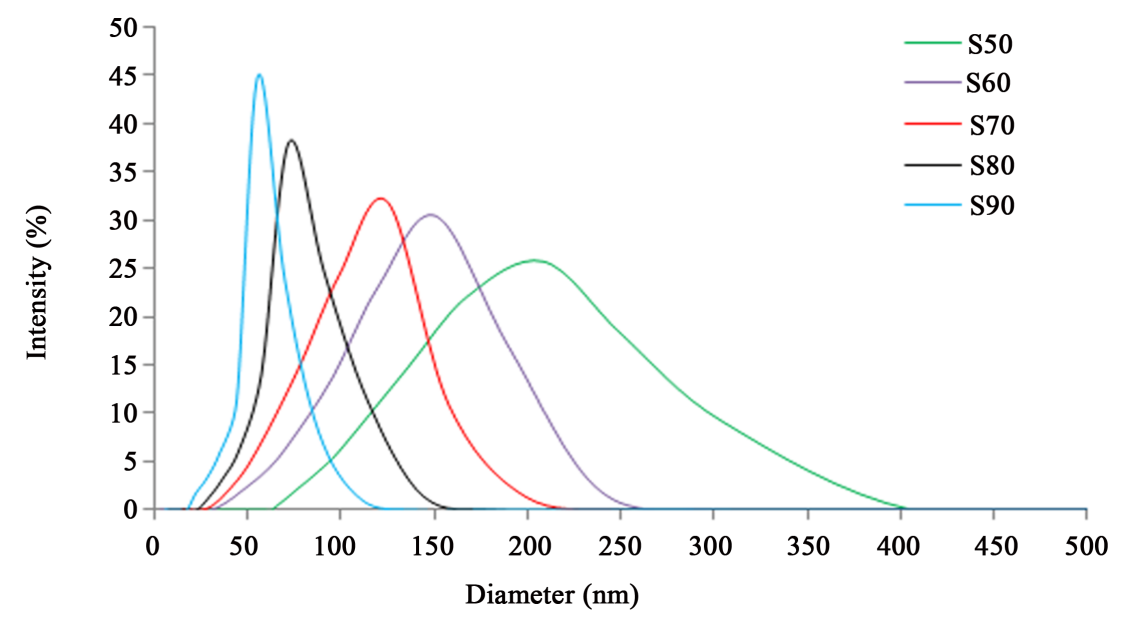

Figure 5. DLS measurement of maghemite nanoparticles suspensions for samples.

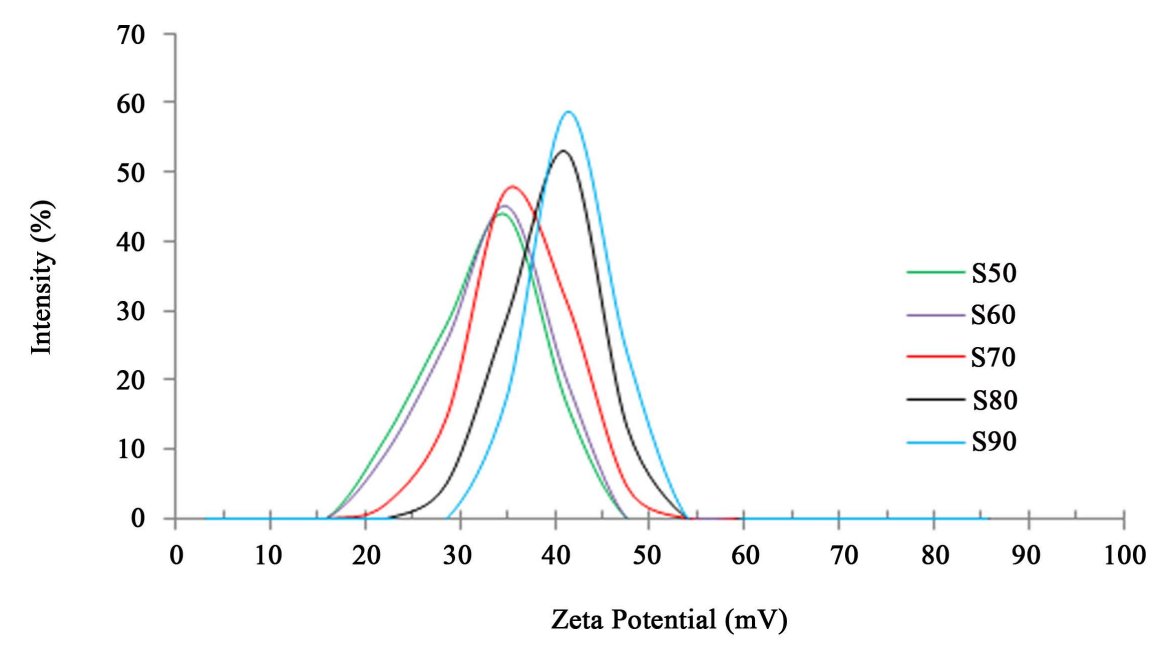

Figure 6. Zeta potential of maghemite nanoparticles suspensions samples. 


\section{Acknowledgements}

This work was funded by Directorate General of Higher Education, Ministry of Research, Technology, and Higher Education of the Republic of Indonesia under Fundamental Research Project no. 047/PL20/R8/SP2-PF/ PL/2015.

\section{References}

[1] Abareshi, M., Goharshadi, E.K., Zebarjad, S.M., Fadafan, H.K. and Youssefi, A. (2010) Fabrication, Characterization and Measurement of Thermal Conductivity of $\mathrm{Fe}_{3} \mathrm{O}_{4}$ Nanofluids. Journal of Magnetism and Magnetic Materials, 322, 3895-3901. http://dx.doi.org/10.1016/j.jmmm.2010.08.016

[2] Sundar, L.S., Singh, M.K. and Sousa, A.C. (2013) Investigation of Thermal Conductivity and Viscosity of $\mathrm{Fe}_{3} \mathrm{O}_{4} \mathrm{Na}^{-}$ nofluid for Heat Transfer Applications. International Communications in Heat and Mass Transfer, 44, 7-14. http://dx.doi.org/10.1016/j.icheatmasstransfer.2013.02.014

[3] Tang, S.C. and Lo, I.M. (2013) Magnetic Nanoparticles: Essential Factors for Sustainable Environmental Applications. Water Research, 47, 2613-2632. http://dx.doi.org/10.1016/j.watres.2013.02.039

[4] Rosensweig, R., Hirota, Y., Tsuda, S. and Raj, K. (2008) Study of Audio Speakers Containing Ferrofluid. Journal of Physics: Condensed Matter, 20, 204147. http://dx.doi.org/10.1088/0953-8984/20/20/204147

[5] Nkurikiyimfura, I., Wang, Y. and Pan, Z. (2013) Heat Transfer Enhancement by Magnetic Nanofluids-A Review. Renewable and Sustainable Energy Reviews, 21, 548-561. http://dx.doi.org/10.1016/j.rser.2012.12.039

[6] Wang, Y., Cao, X., Liu, G., Hong, R., Chen, Y., Chen, X., Li, H., Xu, B. and Wei, D. (2011) Synthesis of $\mathrm{Fe}_{3} \mathrm{O}_{4} \mathrm{Mag}^{-}$ netic Fluid Used for Magnetic Resonance Imaging and Hyperthermia. Journal of Magnetism and Magnetic Materials, 323, 2953-2959. http://dx.doi.org/10.1016/j.jmmm.2011.05.060

[7] Li, Q. and Xuan, Y.M. (2009) Experimental Investigation on Heat Transfer Characteristics of Magnetic Fluid Flow around a Fine Wire under the Influence of an External Magnetic Field. Experimental Thermal and Fluid Science, 33, 591-596. http://dx.doi.org/10.1016/j.expthermflusci.2008.12.003

[8] Huminic, G., Huminic, A., Morjan, I. and Dumitrache, F. (2011) Experimental Study of the Thermal Performance of Thermosyphon Heat Pipe Using Iron Oxide Nanoparticles. International Journal of Heat and Mass Transfer, 54, 656-661. http://dx.doi.org/10.1016/j.ijheatmasstransfer.2010.09.005

[9] Correa, J.R., Canetti, D., Castillo, R., Llópiz, J.C. and Dufour, J. (2006) Influence of the Precipitation pH of Magnetite in the Oxidation Process to Maghemite. Materials Research Bulletin, 41, 703-713. http://dx.doi.org/10.1016/j.materresbull.2005.10.009

[10] Oh, J.K. and Park, J.M. (2011) Iron Oxide-Based Superparamagnetic Polymeric Nanomaterials: Design, Preparation, and Biomedical Application. Progress in Polymer Science, 36, 168-189. http://dx.doi.org/10.1016/j.progpolymsci.2010.08.005

[11] Bee, A., Massart, R. and Neveu, S. (1995) Synthesis of Very Fine Maghemite Particles. Journal of Magnetism and Magnetic Materials, 149, 6-9. http://dx.doi.org/10.1016/0304-8853(95)00317-7

[12] Schwegmann, H., Feitz, A.J. and Frimmel, F.H. (2010) Influence of the Zeta Potential on the Sorption and Toxicity of Iron Oxide Nanoparticles on S. cerevisiae and E. coli. Journal of Colloid and Interface Science, 347, 43-48. http://dx.doi.org/10.1016/j.jcis.2010.02.028

[13] Xu, J., Yang, H., Fu, W., Du, K., Sui, Y., Chen, J., Zeng, Y., Li, M. and Zou, G. (2007) Preparation and Magnetic Properties of Magnetite Nanoparticles by Sol-Gel Method. Journal of Magnetism and Magnetic Materials, 309, 307-311. http://dx.doi.org/10.1016/j.jmmm.2006.07.037

[14] Hsieh, T.-H., Ho, K.-S., Bi, X., Han, Y.-K., Chen, Z.-L., Hsu, C.-H. and Chang, Y.-C. (2009) Synthesis and Electromagnetic Properties of Polyaniline-Coated Silica/Maghemite Nanoparticles. European Polymer Journal, 45, 613-620. http://dx.doi.org/10.1016/j.eurpolymj.2008.12.039

[15] Maleki, H., Simchi, A., Imani, M. and Costa, B. (2012) Size-Controlled Synthesis of Superparamagnetic Iron Oxide Nanoparticles and Their Surface Coating by Gold for Biomedical Applications. Journal of Magnetism and Magnetic Materials, 324, 3997-4005. http://dx.doi.org/10.1016/j.jmmm.2012.06.045

[16] Vidal-Vidal, J., Rivas, J. and Lopez-Quintela, M. (2006) Synthesis of Monodisperse Maghemite Nanoparticles by the Microemulsion Method. Colloids and Surfaces A: Physicochemical and Engineering Aspects, 288, 44-51. http://dx.doi.org/10.1016/j.colsurfa.2006.04.027

[17] Chin, A.B. and Yaacob, I.I. (2007) Synthesis and Characterization of Magnetic Iron Oxide Nanoparticles via w/o Microemulsion and Massart's Procedure. Journal of Materials Processing Technology, 191, 235-237. http://dx.doi.org/10.1016/j.jmatprotec.2007.03.011 
[18] Asuha, S., Suyala, B., Siqintana, X. and Zhao, S. (2011) Direct Synthesis of $\mathrm{Fe}_{3} \mathrm{O}_{4}$ Nanopowder by Thermal Decomposition of Fe-Urea Complex and Its Properties. Journal of Alloys and Compounds, 509, 2870-2873. http://dx.doi.org/10.1016/j.jallcom.2010.11.145

[19] Peng, S., Wang, C., Xie, J. and Sun, S. (2006) Synthesis and Stabilization of Monodisperse Fe Nanoparticles. Journal of the American Chemical Society, 128, 10676-10677. http://dx.doi.org/10.1021/ja063969h

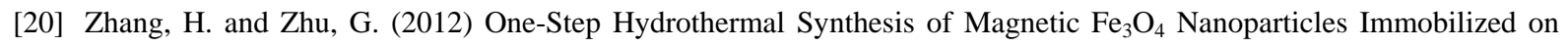
Polyamide Fabric. Applied Surface Science, 258, 4952-4959. http://dx.doi.org/10.1016/j.apsusc.2012.01.127

[21] Caparrós, C., Benelmekki, M., Martins, P.M., Xuriguera, E., Silva, C.J.R., Martinez, L.M. and Lanceros-Méndez, S. (2012) Hydrothermal Assisted Synthesis of Iron Oxide-Based Magnetic Silica Spheres and Their Performance in Magnetophoretic Water Purification. Materials Chemistry and Physics, 135, 510-517. http://dx.doi.org/10.1016/j.matchemphys.2012.05.016

[22] Behdadfar, B., Kermanpur, A., Sadeghi-Aliabadi, H., del Puerto Morales, M. and Mozaffari, M. (2012) Synthesis of Aqueous Ferrofluids of $\mathrm{ZnxFe}_{3}-\mathrm{xO}_{4}$ Nanoparticles by Citric Acid Assisted Hydrothermal-Reduction Route for Magnetic Hyperthermia Applications. Journal of Magnetism and Magnetic Materials, 324, 2211-2217. http://dx.doi.org/10.1016/j.jmmm.2012.02.034

[23] Odenbach, S. (2004) Ferrofluids: Magnetically Controllable Fluids and Their Applications. Applied Rheology, 14, 179179.

[24] Teja, A.S. and Koh, P.-Y. (2009) Synthesis, Properties, and Applications of Magnetic Iron Oxide Nanoparticles. Progress in Crystal Growth and Characterization of Materials, 55, 22-45. http://dx.doi.org/10.1016/j.pcrysgrow.2008.08.003

[25] Kluchova, K., Zboril, R., Tucek, J., Pecova, M., Zajoncova, L., Safarik, I., Mashlan, M., Markova, I., Jancik, D. and Sebela, M. (2009) Superparamagnetic Maghemite Nanoparticles from Solid-State Synthesis-Their Functionalization towards Peroral MRI Contrast Agent and Magnetic Carrier for Trypsin Immobilization. Biomaterials, 30, $2855-2863$. http://dx.doi.org/10.1016/j.biomaterials.2009.02.023

[26] Singh, B.P., Menchavez, R., Takai, C., Fuji, M. and Takahashi, M. (2005) Stability of Dispersions of Colloidal Alumina Particles in Aqueous Suspensions. Journal of Colloid and Interface Science, 291, 181-186. http://dx.doi.org/10.1016/j.jcis.2005.04.091 